\title{
The heterogeneity of meningioma revealed by multiparameter analysis: infiltrative and non-infiltrative clinical phenotypes
}

\author{
EMMANUEL GAY ${ }^{1}$, ELODIE LAGES ${ }^{2}$, CLAIRE RAMUS ${ }^{2}$, AUDREY GUTTIN ${ }^{2,3}$, \\ MICHÈLE EL ATIFI ${ }^{2,3}$, ISABELLE DUPRÉ ${ }^{2,3}$, ALI BOUAMRANI ${ }^{2}$, CAROLINE SALON $^{4}$, \\ DAVID RATEL $^{2}$, DIDIER WION ${ }^{2}$, FRANÇOIS BERGER ${ }^{2}$ and JEAN-PAUL ISSARTEL ${ }^{2,3,5}$ \\ ${ }^{1}$ Department of Neurosurgery, Centre Hospitalier Universitaire; ${ }^{2}$ Grenoble Institut des Neurosciences, \\ INSERM U836 Team 7 Nanomedicine and Brain, Université Joseph Fourier; ${ }^{3}$ Clinical Transcriptomics \\ and Proteomics Platform, Centre Hospitalier Universitaire and Grenoble Institut des Neurosciences; \\ ${ }^{4}$ Department of Pathology, Centre Hospitalier Universitaire, Grenoble; ${ }^{5} \mathrm{CNRS}$, France
}

Received November 2, 2010; Accepted December 23, 2010

DOI: 10.3892/ijo.2011.944

\begin{abstract}
Tumor invasion or infiltration of adjacent tissues is the source of clinical challenges in diagnosis as well as prevention and treatment. Among brain tumors, infiltration of the adjacent tissues with diverse pleiotropic mechanisms is frequently encountered in benign meningiomas. We assessed whether a multiparametric analysis of meningiomas based on data from both clinical observations and molecular analyses could provide a consistent and accurate appraisal of invasive and infiltrative phenotypes and help determine the diagnosis of these tumors. Tissue analyses of 37 meningiomas combined enzyme-linked immunosorbent assay (ELISA) and surface-enhanced laser desorption/ionization time-of-flight (SELDI-TOF) assays of two different protein biomarkers (thrombospondin 1 and a phosphorylated form of vimentin) as well as gene expression analyses with oligonucleotide microarrays. Up to four different clinical and molecular parameters were then examined for tumor classification. From this study, we were able to cluster 36 out of the 37 tumors into two different subsets corresponding to infiltrative/invasive and non-infiltrative tumors. In addition, meningiomas that invade brain and those that infiltrate the neighboring skull bone exhibited no distinguishable molecular features. Our multiparameter analysis that combines clinical data, transcriptomic and molecular assays clearly reveals the heterogeneity of meningiomas and distinguishes the intrinsically infiltrative/invasive tumors from the non-infiltrative meningiomas.
\end{abstract}

Correspondence to: Dr Jean-Paul Issartel, Grenoble Institut des Neurosciences, Centre de Recherche INSERM U836 UJF-CEA-CHU, Equipe 7, Bâtiment Edmond J Safra, Chemin Fortuné Ferrini, 38700 La Tronche, France

E-mail: jean-paul.issartel@ujf-grenoble.fr

Key words: microarray gene profiling, molecular characterization, protein mass spectrometry, thrombospondin 1, tumor infiltration, tumor invasion

\section{Introduction}

Phenotypic characterization of tumors to provide cellular identification of neoplastic tissues is a question of primary clinical interest, but other important intrinsic tumor characteristics should also be considered when designing the most efficient therapeutic strategy: tumor drug sensitivity, tendency to recurrence, propensity to invade vicinal tissues, and ability to generate metastases. Methods to satisfactorily assess tumor features are not currently available or they are not sufficiently accurate for all tumors. Clearly, a molecular description of the cellular components or events that are responsible for these characteristics would be valuable.

In the present study, we provide a molecular characterization of meningiomas, one of the most common central nervous system tumors that arises from arachnoid cells. Driven by unknown cellular determinants, even though they are usually slow-growing tumors and even in their low-grade status, meningiomas often exhibit (in $\sim 20 \%$ of the cases in our experience) a variety of general aggressive behaviors such as penetration of surrounding tissues, modification of bone structure, rapid regrowth of partially resected tumor and recurrence. Tissue infiltrations can target different anatomic areas or adjacent tissues such as the sinuses, the adjacent brain, or the skull bone, thereby causing major focally limited or extensive damage (1-5). Typically, the term invasion is restricted to infiltration into adjacent brain cortex. Since brain infiltration increases the recurrence risk of grade I meningiomas, it has been suggested that brain-infiltrative meningiomas be classified as grade II meningiomas (6). However, some grade I meningiomas may also extensively invade the bone or the sinuses with higher recurrence rates and a shorter patient survival time.

According to the World Health Organization (WHO) grading scale, meningiomas are currently classified into three main grades: grade I (benign tumors, which are the most frequent, accounting for $\sim 94 \%$ of meningiomas), grade II (atypical meningiomas), and grade III (anaplastic tumors) (4,6-8). Several groups have already compared the three different grades meningiomas and/or the various grades of meningiomas versus normal 
brain by gene expression profiling studies (9-13). These studies pinpointed several genes, revealed by their altered expression levels, which are involved in the events controlling meningioma pathogenesis or malignancy progression. However, none of these studies were specifically designed to characterize the invasive phenotypes of these tumors.

Two published investigations reported approaches and identified markers for predicting aggressive meningioma phenotype. In these studies, meningiomas were classified as clinically aggressive by combining diverse criteria such as their histological features (Ki-67 proliferative index, number of mitoses), recurrence rate and clinical signs (tissue invasion, perilesional edema). It was shown that qRT-PCR of c-Myc transcript (14) or fluorescence in situ hybridization (FISH) experiments and ${ }^{1} \mathrm{H}$ magnetic resonance spectroscopy (15) could help distinguish subsets of aggressive versus non-aggressive meningiomas. Moreover, based on an immunohistolabeling approach, SPARC, one extracellular matrix protein was found at a high level in benign, atypical, or malignant meningiomas that shared invasive phenotypes (16).

The present study proposes a molecular characterization of benign meningiomas with a selective and specific focus on their infiltrative/invasive or non-infiltrative phenotypes. Experiments were conducted using different low- or highthroughput technical approaches so as to assay and analyze sets of proteins and transcriptomic biomarkers in 37 meningiomas that exhibited either non-infiltrative phenotypes or on the contrary apparent infiltrative/invasive behaviors, as evidenced by their location in different adjacent tissues or areas such as brain cortex, bone and sinuses. This study had two specific goals. First, it attempted to evaluate whether meningiomas could be subtyped according to their infiltrative/invasive or non-infiltrative features. Second, we aimed to validate the proof of concept of a multiparameter analysis based on molecular markers and/or a combination of different technical approaches for an accurate diagnosis and prognosis. This analysis demonstrated tumor clustering that is quite closely related to the infiltrative/invasive features of the tumor and validated the concept of a multiparameter-based analysis to improve the accuracy of meningioma diagnosis.

\section{Materials and methods}

Clinical features and pathology analysis of the tumors. The study was approved by the local ethics committee and written informed consent was obtained from the patients or their families. Pathological and clinical features were determined in a cohort of 37 randomly chosen patients with meningiomas. This cohort was defined by the average age of the patients, 59 years (range, 34-79), and by a sex ratio of 29 females to eight males (this gender bias was related to the pronounced female prevalence of the pathology). Tumors were graded according to the WHO classification criteria and the recommendations concerning atypical meningiomas (4,6-7). In addition, preoperative magnetic resonance (MR) and computed tomography (CT) scanner imaging as well as the pathology examination reports were reviewed to establish whether the tumors had a non-infiltrative behavior or on the contrary exhibited pathological manifestations of infiltration into adjacent tissues. Tumor features such as sinus or bone infiltration were routinely detected on images. Diagnosis and tissue infiltration were ascertained by pathology examination on paraffin-embedded tissue samples. Brain invasion was ascertained by histological evidence of the presence of meningioma cells inside brain tissue. Hyperostosis or osteolysis with presence of meningioma cells inside bone interstices on pathology examinations were both signs of bone infiltration phenotypes. Meningioma infiltrations of cavernous sinus or veinous sinuses were confirmed by pathology examination searching for the presence of meningioma cells inside adjacent tissues when it was possible to do so, since pathology examinations were most often carried out on resected samples from outside the sinuses. According to these criteria, infiltrative tumors could have pathological manifestations such as a simple infiltration into one tissue or a combined infiltration into several types of neighboring tissues.

We annotated the infiltrative/invasive tumors by specifying whether brain cortex, bone, or sinuses were affected by an infiltration phenomenon. All the clinical data (WHO grade, location, tissue infiltration and recurrence status) are reported Table IA.

Based on the above-mentioned criteria, 33 tumors were classified as grade I meningiomas and four as grade II because these cases showed brain invasion. A total of 20 meningiomas were clinically infiltrative or invasive (Table IA, samples 1-11; 13 ; 15-21 and 25). Brain invasion was found in combination with bone and sinus infiltration in three out of the four brain invasion cases. Bone infiltration with sinus infiltration was observed in ten cases and six tumors exhibited sinus infiltration only.

Tissue samples. The study was approved by the ethics committee. Written consent was obtained from the patients or their families. A total of 37 meningioma samples were collected from the operating room and were immediately frozen and stored at $-80^{\circ} \mathrm{C}$.

Primary culture of meningiomas. Immediately upon surgical removal, tissue samples were chopped into small pieces and placed in Dulbecco's modified Eagle's medium (DMEM) (Invitrogen, Gibco, Cergy-Pontoise, France) supplemented with $10 \%$ fetal calf serum (Abcys, Paris, France), $100 \mathrm{U} / \mathrm{ml}$ penicillin $\mathrm{G}$ and $100 \mu \mathrm{g} / \mathrm{ml}$ streptomycin (Life Science, CergyPontoise, France) to obtain primary cultures. Adherent tumor cells were allowed to grow at $37^{\circ} \mathrm{C}$ in a $5 \% \mathrm{CO}_{2}$ atmosphere.

THBS1 immunocytochemical detection. The immunocytochemical analysis was performed after permeabilization of the cells with Triton X-100 (0.3\%) in PBS, BSA (1.5\%). Permeabilized cells were incubated with a mouse monoclonal anti-human THBS1 antibody (Boehringer/Roche, Meylan, France) at dilution 1:500 in PBS for $4 \mathrm{~h}$ at room temperature. Anti-THBS1 reactivity was detected after incubation with an FITC-labeled secondary antibody (rabbit anti-mouse IgG, AEC Vector) (at dilution 1:1000 for $45 \mathrm{~min}$ at room temperature). Nuclei were stained with propidium iodide.

ELISA determination of THBS1. Cultured cells were incubated for $24 \mathrm{~h}$ in serum-free DMEM and THBS1 concentration was measured by ELISA in culture media following the procedure described by Tenan et al (17). THBS1 concentrations were expressed in ng of THBS1 per mg of total proteins in medium. 


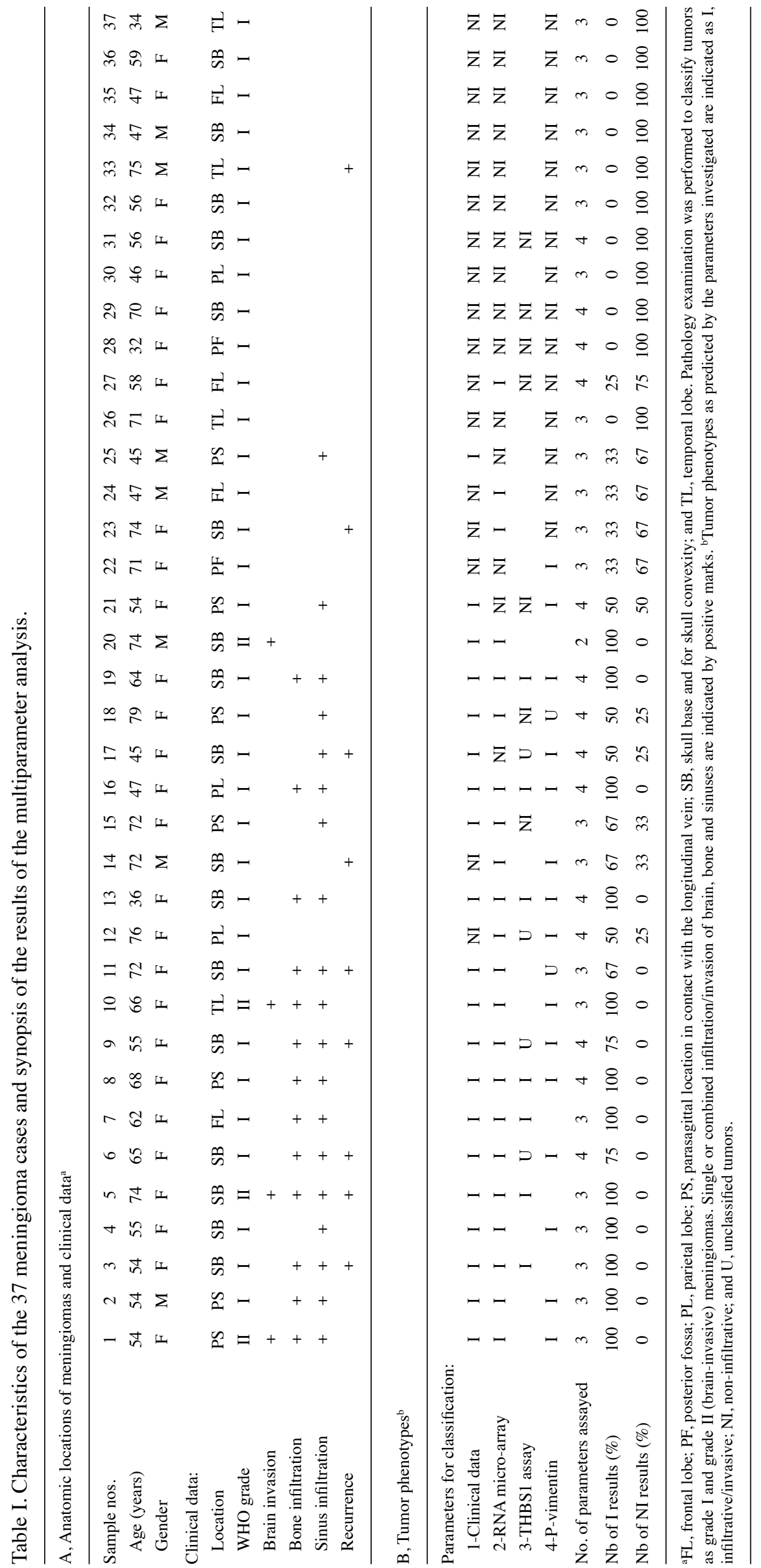



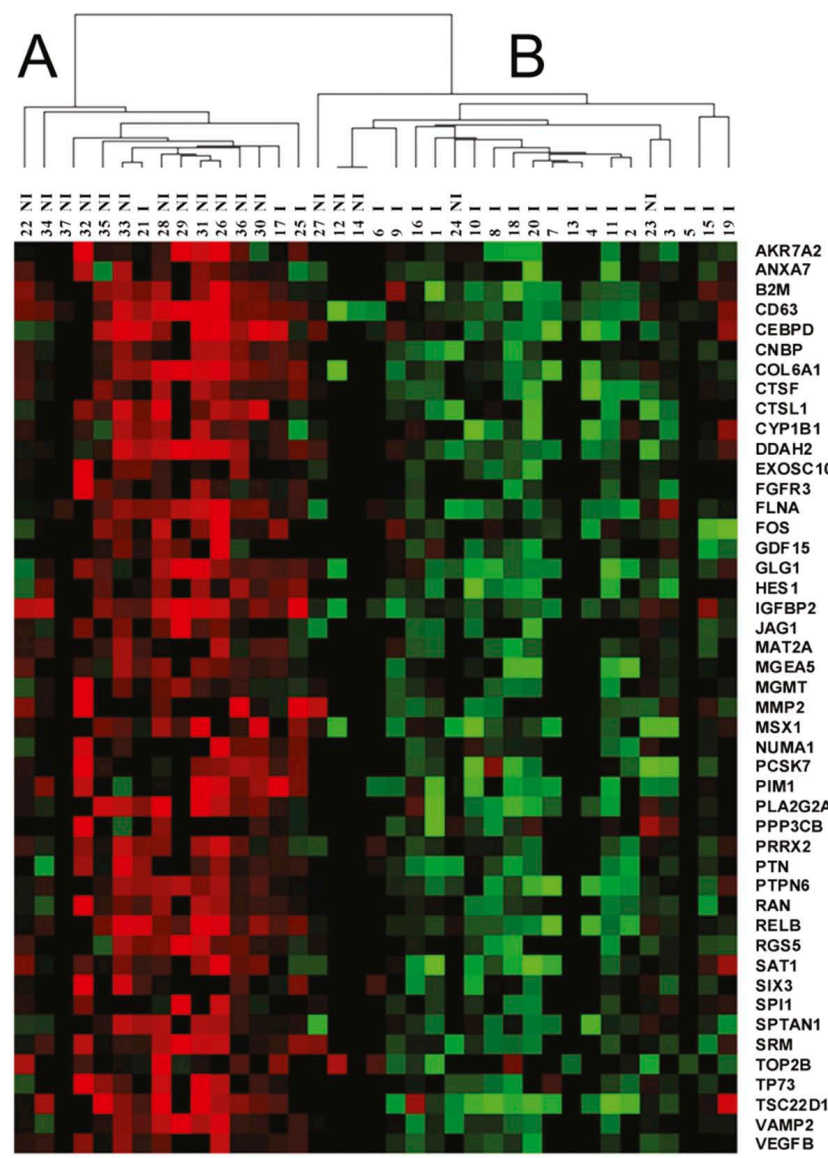

Figure 1. Unsupervised hierarchical clustering of the tumors. A two-dimensional hierarchical clustering of 37 meningiomas (20 infiltrative/invasive and 17 non-infiltrative meningiomas, according to clinical and pathology analysis criteria) was performed with data from the transcriptomic analysis carried out by hybridization with membrane arrays. Discriminant genes (a total of 46) and patient samples were clustered using complete linkage clustering methods from Eisen's cluster software. Clustered trees are displayed using Treeview software. Red squares denote a high expression level and green squares a low expression level in comparison to the mean. The sample numbers and the clinical status of the tumors are indicated (I stands for infiltrative/invasive; NI for non-infiltrative). Gene symbols are reported on the right (see identity in Table II).
All the measurements were taken twice on early cultures (P3 passages) with four determinations for each culture dish.

SELDI-TOF mass spectrometry profiling of tumor lysates. Cryostat slices (10 $\mu \mathrm{m}$ thick) of the frozen tumor specimens were suspended in $300 \mu 1$ of Reporting lysis buffer (Promega, Madison, WI, USA) containing a mixture of antiproteases (Boehringer/Roche), to obtain a final protein concentration close to $2 \mu \mathrm{g} / \mu \mathrm{l}$. After $30 \mathrm{~min}$ of incubation on ice and centrifugation $\left(10,000 \mathrm{x} \mathrm{g}\right.$ for $10 \mathrm{~min}$ at $\left.4^{\circ} \mathrm{C}\right)$, the supernatant was diluted in a binding buffer (100 mM Tris and $0.1 \%$ Triton $\mathrm{X}-100$ at $\mathrm{pH} 8.0$ ) to a final protein concentration of $0.1 \mu \mathrm{g} / \mu \mathrm{l}$ and $100 \mu \mathrm{l}$ of this suspension was applied to Q10 anion-exchange active binding surfaces of SELDI ProteinChip Arrays (Bio-Rad, MarnesLa-Coquette, France). The arrays were washed three times with the binding buffer, then once with the binding buffer without Triton, for $5 \mathrm{~min}$, then once with $2 \mathrm{mM}$ HEPES at $\mathrm{pH}$ 7.5. Sinapinic acid was used as the ionization matrix and ProteinChip arrays were analyzed for high mass range optimization in a Bio-Rad PCS4000 mass spectrometer. The spectra were calibrated using a mixture of standard proteins (Bio-Rad all-in-one standard) and intensity normalization to total ion current in the 4-100-kDa range was carried out with the Biomarker Wizard software (Bio-Rad). The 53-kDa protein peak surface was measured for all the samples.

Transcriptomic analysis. Tissue slices $(40 \mu \mathrm{m}$ thick, a total of approximately $100 \mathrm{mg}$ of tissue) were immediately resuspended in the denaturing solution of the RNA-gents Total RNA isolation system (Promega). Total RNAs were then purified by phenol-chloroform-based extraction following the recommendations of the RNA isolation system (Promega). Quantification and quality control of the extracted RNA samples were assessed by spectrometric measurement and electrophoretic profiling using RNA 6000 Nano kit on a BioAnalyser 2100 (Agilent Technologies, Palo Alto, CA, USA).

cDNAs were obtained by reverse transcription of the total RNA samples using the M-MLV reverse transcriptase (Promega, Charbonnières-les-Bains, France) with poly-dT priming
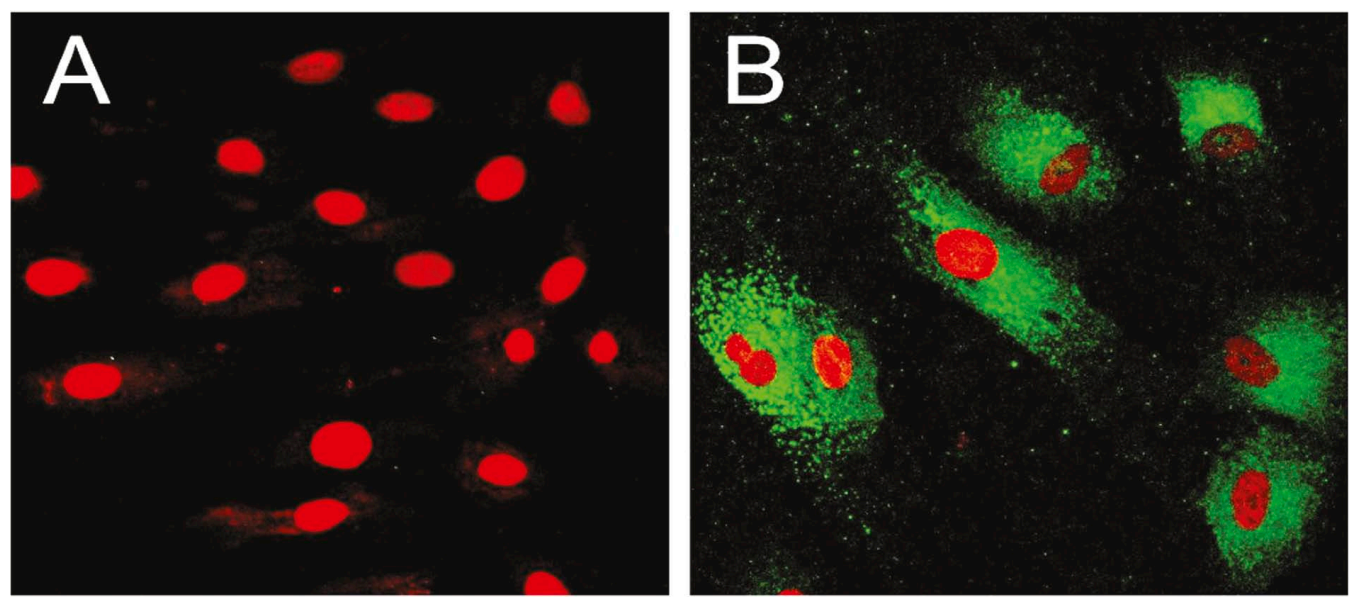

Figure 2. Immunocytochemical staining of primary cultured cells of infiltrative/invasive and non-infiltrative samples for THBS1 detection. Cultured cells were stained with antibodies directed against THBS1 (green labeling) and nuclei were stained with propidium iodide (red). All procedures are described in Materials and methods. (A) Sample from non-infiltrative meningioma. (B) Sample from infiltrative meningioma. 


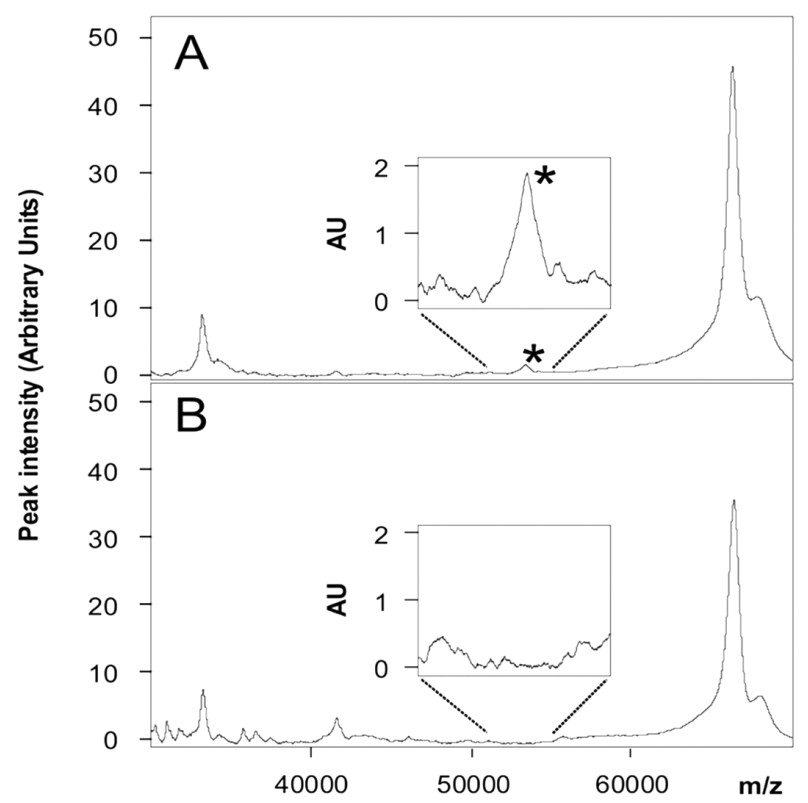

Figure 3. SELDI-TOF protein profiles using Q10 anion-exchange ProteinChip arrays. The protein mass spectra correspond to the analysis of tissue lysates from either a non-infiltrative (A) or an infiltrative tumor (B). Inset, close-up of the phosphorylated vimentin (*) that is detectable in the non-infiltrative tumor samples.

and $\alpha^{33} \mathrm{P}$-dCTP (18). Radiolabeled cDNAs were purified by column filtration (Microcon YM-50, Millipore, Saint Quentinen-Yvelines, France) and hybridized to membrane arrays. Membrane arrays were prepared as described previously (18) by spotting in duplicate 70 -mer oligonucleotides onto nylon membranes (Hybond N+, Amersham, Velizy, France) using an Affymetrix R417 Microarrayer (MWG, Ebersberg, Germany). The set of oligonucleotides was chosen to allow detection of transcripts corresponding to 800 genes with expected roles in oncogenesis, as reported in the literature (19).

After hybridization and washing, membrane arrays were exposed to a high-resolution phosphor screen for 3 days and then scanned (BAS-5000, Fuji-Film; Raytest, Paris la Defense, France). Recorded signals were quantified using the Array Gauge Software (Fuji-Film). Only transcripts detected by spot intensities higher than three times the value of the background average intensity were kept for further analysis. Inter-assay data were normalized with the global normalization method using the linear regression of all intensity data of the arrays. Expression levels were reported in arbitrary units. The relative changes in expression status for all the genes and different samples were analyzed using the hierarchical Cluster and Treeview software packages available on http://bonsai. hgc.jp/ mdehoon/software/cluster/software.htm.

\section{Results}

Membrane-array-based transcriptomic analysis. Gene expression profiling was carried out for all the 37 tumor samples. This was done using homemade membrane arrays that allowed us to investigate the individual expression levels of 800 different genes of general interest in oncology. For most of the 800 analyzed genes, the expression levels were found to be either undetectable or expressed at the same level in all the meningioma samples.
An unsupervised hierarchical clustering of the samples was built with the expression data of 46 genes for which changes in the levels of expression in several tumors were noticeable (Materials and methods). The resulting clustering pattern (Fig. 1) sorted the 37 tumors into two main groups (clusters A and B, Fig. 1) based on these 46 genes with significant differential expression levels between the two groups of tumors $(\mathrm{p}<0.05)$ (Table II). Group A clustered 15 tumors, most of them with a non-infiltrative/noninvasive phenotype (12/15). Group B grouped 22 samples, with most of them corresponding to tumors with infiltrative/invasive phenotypes according to the clinical and pathology criteria $(17 / 22)$. It is worth noting that the clustering of the 37 tumors only evidenced downregulated genes in cluster B samples in comparison to cluster A samples. From the available information of the gene ontology functions, the differentially expressed genes encode either enzymes and structural proteins, receptors and proteins involved in signal transduction or gene products with potential functions in DNA binding and transcription (Table II). For several of these pinpointed genes, in the context of diverse specific tumors, downregulation has been correlated with increased invasiveness, tumor progression, or decreased apoptosis. This was already reported for ANXA7 (involved in calcium signaling) (20); $C D 63$ (involved in signal transduction) (21); CEBPD, FOS, HES1, MSX1, TP73 and TSC22D1 (all playing a role at least in transcription) (22-27); GLGl (fibroblast growth factor receptor) (28); PLA2G2A (phospholipase activity) (29) and PTPN6 (protein tyrosine phosphatase activity) (30). These data may support the idea that a decreased expression of these genes might be consistent with cellular events that are relevant with the infiltrative behaviors of meningiomas.

Immunocytochemical detection and ELISA assay of THBSI in cultured cells. Since it has been already established that SPARC, an extracellular matrix protein, is a candidate marker for invasiveness and is overexpressed in invasive tumors (16), we decided to have a closer look at the amounts of another specific extracellular matrix protein, thrombospondin 1 (THBS1). So we analyzed the level of expression of this protein by immuno-based assays. Immunocytochemical analysis of cultured meningioma cells revealed that there were very different levels of THBS1 concentrations in the cultured samples. Most of the time, the highest amount of THBS1 was found in cultured cells from meningiomas with an infiltrative/invasive phenotype (Fig. 2). Data reported in the literature (31), reinforced by our immunocytochemical pictures of the cultured meningioma cells, suggested that THBS1 is secreted in the surrounding medium. We then assayed the amount of THBS1 in the growth medium of cultured meningioma cells using ELISA. In primary cultures, cells from meningiomas classified as infiltrative/invasive tumors based on their clinical features were found to secrete larger amounts of THBS1 (mean, $1740 \mathrm{ng} / \mathrm{mg}$ of total proteins) than cells from noninfiltrative tumors (mean, $29 \mathrm{ng} / \mathrm{mg}$ of total proteins).

SELDI-TOF mass spectrometry profiles and correlation with THBS1 data. SELDI-TOF mass spectrometry was used to analyze protein complexity in meningioma tissue lysates. This approach helped us to identify in the mass spectrometry profiles a peak corresponding to a $53-\mathrm{kDa}$ molecular mass protein that was detectable in most of the non-infiltrative tumors but absent in the infiltrative/invasive tumors (Fig. 3). 
Table II. Biological identity of the genes with discriminative expression between infiltrative/invasive and non-infiltrative meningiomas. ${ }^{\mathrm{a}}$

\begin{tabular}{ll} 
Gene symbol & \multicolumn{1}{c}{ Full names } \\
\hline AKR7A2 & $\begin{array}{l}\text { Aldo-keto reductase family 7, member A2 } \\
\text { (aflatoxin aldehyde reductase) }\end{array}$ \\
ANXA7 & Annexin A7 \\
B2M & $\begin{array}{l}\text { B-2-microglobulin } \\
\text { CD63 }\end{array}$ \\
CD63 molecule \\
CEBPD & CCAAT/enhancer binding protein (C/EBP), $\delta$ \\
CNBP & $\begin{array}{l}\text { Zinc finger protein 9 }=\text { CCHC-type zinc finger, } \\
\text { nucleic acid binding protein }\end{array}$ \\
& $\begin{array}{l}\text { Collagen, type VI, } \alpha 1 \\
\text { COL6A1 }\end{array}$ \\
CTSF & $\begin{array}{l}\text { Cathepsin F } \\
\text { CTSL1 }\end{array}$ \\
CYP1B1 & $\begin{array}{l}\text { Cytochrome P450, family 1, subfamily B, } \\
\text { polypeptide 1 }\end{array}$ \\
&
\end{tabular}

DDAH2 Dimethylarginine dimethylaminohydrolase 2

EXOSC10 Exosome component 10

FGFR3 Fibroblast growth factor receptor 3 (achondroplasia, thanatophoric dwarfism)

FLNA Filamin A, $\alpha$ (actin binding protein 280)

FOS v-fos FBJ murine osteosarcoma viral oncogene homolog

GDF15 Growth differentiation factor 15

GLG1 Golgi apparatus protein 1

HES1 Hairy protein

IGFBP2 Insulin-like growth factor binding protein 2, $36 \mathrm{kDa}$

JAG1 Jagged 1 (Alagille syndrome)

MAT2A Methionine adenosyltransferase II, $\alpha$

MGEA5 Meningioma expressed antigen 5 (hyaluronidase)

Aldehyde reductase activity, electron carrier activity, oxidoreductase activity

Rhodopsin-like receptor activity, calcium ion binding, protein binding, calcium-dependent phospholipid binding

Protein binding

Antigen associated with early stages of melanoma tumor progresion. May play a role in growth regulation.

Transcription factor activity, sequence-specific DNA binding, protein dimerization activity

Nucleic acid binding, single-stranded DNA binding, transcription factor activity, single-stranded RNA binding, protein binding, zinc ion binding, metal ion binding

Structural molecule activity, protein binding, motor activity, ATP binding Cysteine-type endopeptidase activity, cathepsin F activity Cysteine-type endopeptidase activity, cathepsin L activity, protein binding

Monooxygenase activity, iron ion binding, protein binding, oxygen binding, electron carrier activity, heme binding, metal ion binding, unspecific monooxygénase activity

Protein binding, dimethylargininase activity, hydrolase activity, acting on carbon-nitrogen (but not peptide) bonds, in linear amidines, $\mathrm{NF}-\kappa \mathrm{B}$ binding

Nucleic acid binding, RNA binding, protein serine/threonine kinase activity, 3'-5' exonuclease activity, hydrolase activity, identical protein binding Nucleotide binding, protein kinase activity, protein-tyrosine kinase activity, protein serine/threonine kinase activity, receptor activity interleukin-1 receptor activity, ATP binding, fibroblast growth factor activity, transferase activity, identical protein binding

Actin binding, signal transducer activity, protein binding, transcription factor binding, protein kinase $\mathrm{C}$ binding

Double-stranded DNA binding, transcription factor activity, specific RNA polymerase II transcription factor activity, sequence-specific DNA binding, protein heterodimerization activity

Cytokine activity, growth factor activity

Receptor binding, fibroblast growth factor binding

DNA binding, transcription repressor activity, transcription regulator activity

Insulin-like growth factor binding, oxydoreductase activity, insulinlike growth factor I binding, insulin-like growth factor II binding, FAD binding

Notch binding, structural molecule activity, calcium ion binding, protein binding, growth factor activity

Nucleotide binding, magnesium ion binding, methionine adenosyltrans ferase activity, ATP binding, transferase activity, potassium ion binding cobalt ion binding

Histone acetyltransferase activity, hyalurononglucosaminidase activity, $\beta$-N-acetylhexosaminidase activity, acyltransferase activity, transferase activity, hydrolase activity, acting on glycosyl bonds

DNA binding, catalytic activity, methylated-DNA-[protein]-cysteine S-methyltransferase activity, cysteine-type endopeptidase activity, methyltransferase activity, zinc ion binding, DNA-methyltransferase activity, transferase activity, metal ion binding 
Table II. Continued.

Gene symbol Full names $\quad$ GO molecular function term (from Genecards)

MMP2 Matrix metallopeptidase 2 (gelatinase A, $72 \mathrm{kDa}$ gelatinase, $72 \mathrm{kDa}$ type IV collagenase)

MSX1 msh homeobox 1

NUMA1 Nuclear mitotic apparatus protein 1

PCSK7 Proprotein convertase subtilisin/kexin type 7

PIM1 pim-1 oncogene

$\begin{array}{ll}\text { PLA2G2A } & \begin{array}{l}\text { Phospholipase A2, group IIA } \\ \text { (platelets, synovial fluid) }\end{array} \\ \text { PPP3CB } & \begin{array}{l}\text { Protein phosphatase } 3 \text { (formerly 2B), } \\ \text { catalytic subunit, } \beta \text { isoform }\end{array}\end{array}$

PRRX2 Paired related homebox 2

PTN Pleiotrophin

PTPN6 Protein tyrosine phosphatase, non-receptor type 6

RAN RAN, member RAS oncogene family

RELB V-rel reticuloendotheliosis viral oncogene homolog $\mathrm{B}$

RGS5 Regulator of G-protein signaling 5

SAT1 Spermidine N1-acetyltransferase 1

SIX3 SIX homeobox 3

SPI1 Spleen focus forming virus (SFFV) proviral integration oncogene spil

SPTAN1 Spectrin, $\alpha$, non-erythrocytic 1 ( $\alpha$-fodrin)

SRM Spermidine synthase

TOP2B Topoisomerase (DNA) II $\beta 180 \mathrm{kDa}$

TP73

Tumor protein $\mathrm{p} 73$

TSC22D1 TSC22 domain family, member 1

VAMP2 Vesicle-associated membrane protein 2 (synaptobrevin 2)

VEGFB
Catalytic activity, metalloendopeptidase activity, gelatinase A activity, calcium ion binding, protein binding, metallopeptidase activity, zinc ion binding, metal ion binding

DNA binding, transcription factor activity, protein binding, transcription repressor activity, sequence-specific DNA binding

Nucleotide binding, structural molecule activity, protein binding, microtubule binding

Subtilase activity, peptidase activity

Nucleotide binding, protein kinase activity, protein serine/threonine kinase activity, protein tyrosine kinase activity, protein binding, ATP binding, transferase activity, manganese ion binding, metal ion binding

Phospholipase A2 activity, calcium ion binding, hydrolase activity calcium-dependent phospholipase A2 activity

Phosphoprotein phosphatase activity, protein serine/threonine phosphatase activity, iron ion binding, calcium ion binding, calmodulin binding, zinc ion binding, hydrolase activity

DNA binding, transcription factor activity, sequence-specific DNA binding Protein phosphatase inhibitor activity, growth factor activity, heparin binding Phosphotyrosine binding, protein tyrosine phosphatase activity, molecular function, protein binding, hydrolase activity, phosphatase activity

Nucleotide binding, chromatin binding, transcription coactivator activity GTPase activity, signal transducer activity, protein binding, GTP binding guanyl nucleotide binding, androgen receptor binding

Transcription factor activity, transcription corepressor activity, protein binding

Signal transducer activity, GTPase activator activity

Acyltransferase activity, diamine $\mathrm{N}$-acetyltransferase activity

DNA binding, transcription factor activity, RNA polymerase II transcription factor activity, enhancer binding, protein binding, transcription repressor activity, sequence-specific DNA binding

DNA binding, transcription factor activity, RNA binding, protein binding transcription activator activity, sequence-specific DNA binding

Actin binding, structural constituent of cytoskeleton, calcium ion binding calmodulin binding

Catalytic activity, spermidine synthase activity, transferase activity

Nucleotide binding, DNA binding, chromatin binding, DNA topoisomerase (ATP-hydrolyzing) activity, protein kinase $\mathrm{C}$ binding, protein kinase activity, ATP binding, protein C-terminus binding, histone deacetylase binding, protein heterodimerization activity

DNA binding, transcription factor activity, protein binding, zinc ion binding, metal ion binding

Transcription factor activity

Protein binding, SNARE binding, calmodulin binding, phospholipid binding

Vascular endothelial growth factor receptor binding, protein binding, growth factor activity, heparin binding, nutrient reservoir activity

${ }^{\mathrm{a} O f f i c i a l}$ gene symbols are reported with their official full names. Gene ontology molecular function terms were obtained from the Genecards database (http:// www.genecards.org). 


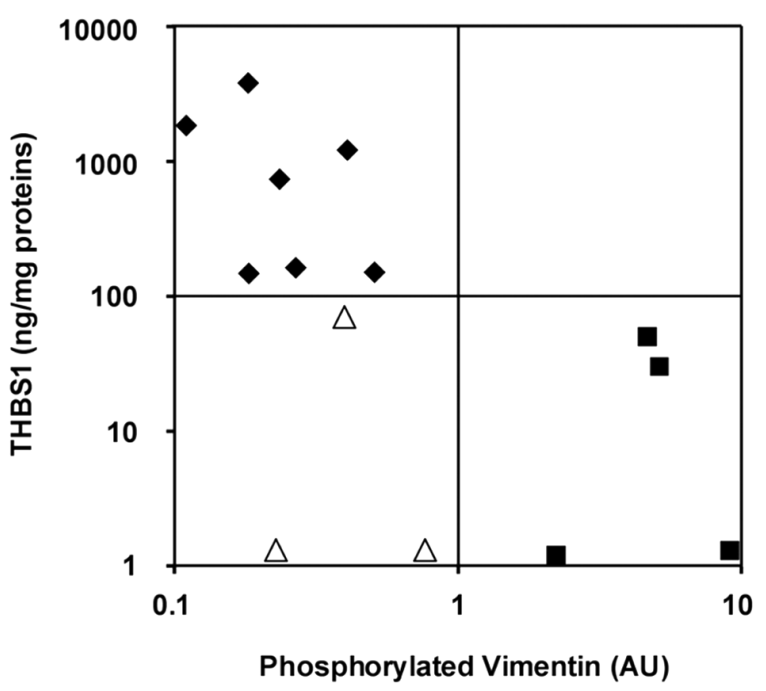

Figure 4. Plot of the amounts of THBS1 in the medium of cultured meningioma cells versus the amounts of the phosphorylated vimentin assessed by SELDI-TOF mass spectrometry in tumor lysates. Assays of THBS1 and of the phosphorylated vimentin were performed as described in Materials and methods. X-axis: total amount of the phosphorylated vimentin assessed in tumor lysates (AU). Y-axis: THBS1 amounts expressed in $\mathrm{ng} / \mathrm{mg}$ of total proteins in the growth medium of cultured meningioma cells from the corresponding tumors. $\mathrm{X}$ and $\mathrm{Y}$ axes are in logarithmic scales. Filled diamonds: samples exhibiting high amounts of THBS1 ( $>100 \mathrm{ng} / \mathrm{mg}$ of total proteins) but low amounts of the phosphorylated vimentin $(<1$ arbitrary unit); filled squares: samples with low amounts of THBS1 $(<100 \mathrm{ng} / \mathrm{mg})$ and high amounts of the phosphorylated vimentin ( $>1$ arbitrary unit); open triangles: samples with low amounts of both THBS1 and phosphorylated vimentin.

This marker was identified as a phosphorylated form of vimentin (32). For quantitative assessment of the amount of the phosphorylated vimentin in 14 tumor samples, the surface of the peak was measured in the spectra. We then plotted the amount of secreted THBS1 by the cultured meningioma cells (see above) versus the amount of the phosphorylated vimentin in the corresponding tumors (Fig. 4). From this figure, three different groups of tumors can be tentatively identified. A first group corresponds to tumors with low amounts of both THBS1 (values lower than $100 \mathrm{ng} / \mathrm{mg}$ of total proteins) and phosphorylated vimentin $(<1 \mathrm{AU})$. The second group includes tumors with a high amount of THBS1 (values higher than $100 \mathrm{ng} / \mathrm{mg}$ of total protein) and a low amount of phosphorylated vimentin. Most of the clinically infiltrative/invasive tumors were found clustered in this group. Finally, the third set groups tumors with a low amount of THBS1 and a high level of phosphorylated vimentin (higher than $1 \mathrm{AU}$ ) that exhibited non-infiltrative features.

Final tumor classification and overall quality assessment of the multiparameter analysis. We attempted to take into account all the different criteria assessed in this study and to validate whether a multiparameter approach can help accurately predict infiltrative/invasive or non-infiltrative phenotypes of all the tumors. A synopsis of all the results and correlations between clinical observations and the different molecular assays was therefore generated (Table IB) according to the following rationale: based on the results of the diverse experiments or clinical data, tumors were labeled infiltrative/invasive (I) or non-infiltrative (NI) or unclassified (U) tumors. In this

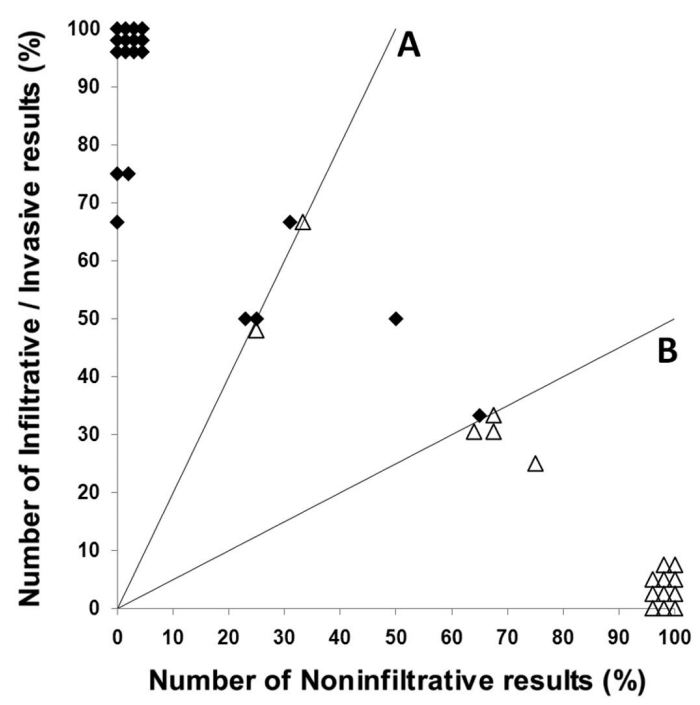

Figure 5. Scatter plot of the samples according to their infiltrative/invasive or non-infiltrative predictable status. The percentage of the total number of assayed parameters that are indicative of an infiltrative/invasive status of the tumors are plotted versus the percentage of the total number of parameters that are indicative of a non-infiltrative status for all the samples (Table I). Filled diamonds: tumors clinically phenotyped as infiltrative/invasive, open diamonds: tumors clinically phenotyped as non-infiltrative. Lines A and B illustrate specific ratios of $\mathrm{y}$-axis values to $\mathrm{x}$-axis values: A line for a ratio of 2; $\mathrm{B}$ line for a ratio of 0.5 .

labeling process, the different molecular assays as well as the clinical data were all taken as four independent parameters for classification (Table I). From the clinical observations and pathology analyses (Table I, parameter 1: clinical data), tumors were classified into only two groups, the non-infiltrative (NI) or the infiltrative/invasive (I) tumor groups. Data from the transcriptomic analysis also generated two main clusters (Fig. 1). Tumors in cluster A (sample numbers 17, 21, 22, 25, 26, 28-37) were called non-infiltrative tumors and tumors in cluster B (sample numbers 1-16, 18-20, 23, 24, 27) were classified as infiltrative/invasive tumors (Table I, parameter 2: RNA micro-array). For all the other molecular assays, tumors were classified as infiltrative/invasive or non-infiltrative regarding the quantitative level of the parameter assessed in comparison to the threshold levels we specified. Tumors with intermediate values for a specific parameter were otherwise labeled unclassified tumors. In the case of the THBS1 ELISA assay (Table I, parameter 3: THBS1 assay), all samples producing $<50 \mathrm{ng} / \mathrm{mg}$ total protein in the medium of in vitro cultured cells were called non-infiltrative tumors, those with values between 50 and $300 \mathrm{ng} / \mathrm{mg}$ total protein were unclassified tumors, and those with values higher than $300 \mathrm{ng} / \mathrm{mg}$ total protein were described as infiltrative/invasive tumors. Finally, tumors with phosphorylated vimentin measured above 2 arbitrary units were labeled non-infiltrative tumors, those with an amount between 0.5 and 2 arbitrary units were labeled unclassified tumors, and those with values lower than 0.5 were considered as infiltrative/invasive tumors.

For each tumor sample, we then calculated the percentage of the total number of available parameters that are indicative of an infiltrative/invasive status as well as the percentage of the parameters that predict a non-infiltrative status. The percent- 
Table III. Parameter accuracy. ${ }^{\text {a }}$

\begin{tabular}{lccc}
\hline Parameter & Approaches & Sensitivity & Specificity \\
\hline Clinical data & Imaging and pathology analysis & 0.90 & 0.94 \\
Transcriptomics & Hybridisation on micro-arrays & 0.95 & 0.81 \\
THBS1 & ELISA & 0.54 & 1.00 \\
Phosphorylated vimentin & SELDI TOF & 0.87 & 0.94 \\
\hline
\end{tabular}

aAll the parameters investigated for diagnosis and the approaches used are indicated. Sensitivity was taken as the proportion of actual infiltrative tumors which are correctly identified as such and specificity as the proportion of non-infiltrative tumors which are correctly identified by a given method.

Table IV. Consistency between parameters and methods. ${ }^{\mathrm{a}}$

\begin{tabular}{lccc}
\hline & Clinical data & Transcriptomics & THBS1 \\
\hline Transcriptomics & $78(29 / 37)$ & & \\
THBS1 & $61(11 / 18)$ & $61(11 / 18)$ & \\
Phosphorylated & $81(26 / 32)$ & $75(24 / 32)$ & $57(8 / 14)$ \\
vimentin & & & \\
\hline
\end{tabular}

${ }^{a}$ The percentage of tumors identically phenotyped by two different parameters or methods are indicated. Parentheses: number of consistent phenotypings/total number of samples phenotyped by either indicated parameters or methods.

ages of infiltrative/invasive parameters were plotted versus the percentage values of non-infiltrative parameters (Fig. 5). In this figure, the clinically diagnosed infiltrative/invasive samples are indicated by filled diamonds and the clinically diagnosed non-infiltrative samples by open diamonds.

At first glance, the 37 cases did not seem to be discriminated into two perfectly separate clusters, one with all the infiltrative/ invasive tumors and another one with the non-infiltrative tumors. However, the ratio between the total number of parameters that predict an invasive status and the total number of parameters that are indicative of a non-invasive status (I/NI ratio, referred to as the infiltrative index) can help delineate distinct areas in Fig. 5. The area left of line A groups samples with an infiltrative index $>2$ (putative infiltrative samples), while the area below line B groups tumors with an infiltrative index $<0.5$ (putative non-infiltrative samples).

According to this distribution based on the $\mathrm{I} / \mathrm{NI}$ infiltrative index, it was possible to identify a total of 20 tumors with potential infiltrative/invasive behaviors (samples 1-20) and 16 other tumors with the non-infiltrative phenotype (samples 22-37) (Table I). Only one tumor with a clinically described infiltrative phenotype (sample 21 in Table I), had an infiltrative index between 0.5 and 2 and therefore was unclassified tumor. Two tumors (cases 12 and 14) with a non-infiltrative phenotype according to the clinical observations were clustered in the group of infiltrative/invasive tumors in the present multiparameter analysis. In addition, in the non-infiltrative cluster, only one clinically diagnosed infiltrative tumor (case number 25) seemed misclassified.
Intrinsic validity of the individual analytical parameters. We also made a comparative evaluation of all the individual parameters or assays regarding their ability to appropriately classify the tumors by calculating the sensitivity and specificity of the assays. Sensitivity was taken as the proportion of actual infiltrative tumors (samples 1-20, as classified from the present multiparameter study) which are correctly identified as such and specificity as the proportion of non-infiltrative tumors (samples 22-37) which are correctly identified by a given method. These results are reported in Table III. Clinical evaluation and phosphorylated vimentin assay appeared valuable individual parameters for infiltrative/non-infiltrative phenotype prediction.

Independently of the final classification of tumor phenotypes, it was also possible to benchmark the different parameters to each other by checking the consistency of the results obtained with two different parameters or assays. Hence, the percentage of tumors identically classified as infiltrative/invasive or noninfiltrative by two parameters are reported in Table IV. The overall consistency between these four criteria ranged from $\sim 57$ to $81 \%$ of the different samples.

\section{Discussion}

The aim of this study was to analyze a cohort of 37 meningioma samples, both at the clinical and molecular levels, in an attempt to investigate whether tumors exhibit clear-cut features that correlate with their non-invasive phenotype or their complex infiltrative/invasive behaviors. This study was also designed to assess the biological assays and clinical observations that have the highest value in providing the most appropriate classification of the different types of meningioma.

To achieve these goals, we carried out a multiparameter analysis of the tumors including four different variables. The first one was the clinical evaluation of the tumor phenotypes, based on tissue examination and combines imaging techniques, surgical observations as well as histological examinations. The other parameters relied on molecular analyses of the tumor samples. One belongs to the field of transcriptomics and is designed to cluster tumors according to the expression levels of 46 genes. The other parameters measure the amounts of two different proteins in the tissue samples or in tumoral cells. A phosphorylated form of vimentin was assessed in tumor lysates using a mass spectrometry-based assay and 
the amount of thrombospondin 1 (THBS1) expressed in the medium of primary cultured tumoral cells was measured by ELISA.

From the synoptic table of the recorded data (Table I) and Fig. 5, tumors were tentatively classified into three different groups (infiltrative/invasive, non-infiltrative and unclassified). For this process, and for every tumor, when the number of assessed parameters that predict an infiltrative/invasive phenotype was at least twice the number of parameters that are indicative of a non-infiltrative phenotype, we decided to classify this tumor as infiltrative/invasive. On the contrary, when the number of parameters that predict an infiltrative/invasive phenotype was found to be half or less than the number of parameters that predict a non-infiltrative phenotype, the tumor was classified as non-infiltrative. This led to the classification of 20 meningiomas (samples 1-20) as infiltrative/invasive tumors and 16 others (samples 22-37) as non-infiltrative (Table I).

The parameters of the current analysis were challenged individually so as to assess which of them were the best classifiers for tumor phenotype characterization. Clinical data, and the phosphorylated vimentin assay, showed reasonable consistency and accurately classified the tumors (Tables III and IV).

It should also be noted that we found that concentrations of the phosphorylated vimentin and THBS1 are inversely correlated in meningiomas according to their phenotypes. Infiltrative/invasive tumors showed high amounts of THBS1 and low amounts of the phosphorylated vimentin, whereas non-infiltrative tumors were characterized by low amounts of THBS1 and high levels of the phosphorylated vimentin. This observation shows that combined use of assays with some markers overexpressed and others underexpressed in tumors should greatly enhance the accuracy of the classification of these tumors.

Our classification of the tumors into the two distinct phenotypic groups should also be critically evaluated. In fact, the data displayed in Fig. 5 show that there seems to be a continuum from the infiltrative/invasive tumors at one extreme to the non-infiltrative ones at the other extreme. As stated above, improvements in the choice of the discriminative markers will probably help sort the different tumors unambiguously. Again, to lower the number of unclassified tumors, it is important to accurately appraise the precise ranges of the threshold values of the parameters that correlate with the distinct behaviors (infiltrative/invasive versus non-infiltrative). This improved assessment of the ranges of values is expected after prospective analysis of large cohorts of patients is conducted.

However, tumors may not be triggered from a non-infiltrative to an infiltrative/invasive phenotype by a single off-and-on phenomenon. Some tumors may have only reached early stages of this phenotype conversion with clinical signs that are not yet detectable, while some or all of the molecular events are already committed in the non-infiltrative to infiltrative conversion. This hypothesis may be supported in the present study by two tumors samples (12 and 14) that show molecular parameters indicative of infiltrative/invasive phenotypes but clinical non-infiltrative signs. On the other hand, in the case of tumor sample 25, which is clinically diagnosed as infiltrative even though it exhibits the molecular parameters of a noninfiltrative tumor, we can assume that the clinical data might have been erroneously or overinterpreted by the clinical staff.
These may be two of the main assets of the multiparameter study: first to rectify misinterpretations and second to unveil original molecular and behavioral stages of the non-infiltrativeto-infiltrative/invasive conversion event. For the practitioner, patients with tumors that show an uncertain phenotype after multiparameter analysis may require very specific care.

Our report as well as others clearly argue for subtyping benign meningiomas on the basis of data that describe meningioma physiopathology at the molecular level in addition to standard histopathology and histomorphological criteria. For example, Pfisterer et al studied a cohort of 30 grade I meningiomas, some of which showed clinically aggressive behaviors (dura or bone infiltration phenotypes, rapid recurrence) and found correlations between chromosomal aberrations exemplified by FISH or metabolite assays by NMR and the aggressive phenotype of the tumors (15).

On the other hand, Durand et al were able to distinguish 36 grade I meningiomas on the basis of the $c M y c$ gene expression level. This subtyping correlated well with the main histopathology criteria such as the Ki-67 proliferative index and the number of mitoses detected by histological examination (14). The conclusions of our investigation carried out on 37 grade I meningioma samples are in agreement with the above-mentioned results and provide evidence that benign meningiomas can be subtyped into two different groups according to their molecular features, with a clear correlation with clinical infiltrative/invasive phenotypes or outcomes. In addition, our study also suggests that there is no apparent molecular distinction between the infiltrative grade I benign tumors and the brain-invasive grade II tumors. The different types of infiltrative/invasive tumors were apparently clustered by our results as a homogeneous group whether or not they infiltrate one or more of the different types of tissues (sinuses, brain cortex and bone). It seems clinically practical to conclude that tumors exhibiting bone, brain cortex, or sinus infiltration features, as evidenced by clinical observations, imaging, or pathology examinations, should be first considered as entities with equivalent potential development behavior and presumably morbidity.

Finally, the molecular rationale that can sustain a possible non-infiltrative to infiltrative/invasive conversion remains to discover. Remarkably, this work identifies THBS1, as an overexpressed protein in infiltrative/invasive tumors. THBS1 is a protein that belongs to the group of matrix-extracellular proteins and has been reported to control adhesion and de-adhesion between cells (33). It is involved in tumor cell migration and invasion, a role that it shares with SPARC, another matrix-extracellular protein that was previously found to be overproduced in invasive meningiomas of the three different grades (16).

In conclusion, we found our technical approach valuable for clinical purposes and we expect that molecular characterization of infiltrative/invasive meningiomas could provide accurate classification, diagnosis and prognostic evaluation of these tumors. This may ultimately help set up useful therapeutic approaches for the control of tissue infiltration by tumors.

\section{Acknowledgments}

We thank Mrs. Danièle Adamski and Professor Basile Pasquier for expert assistance and Dr Linda Northrup for editing the text. 
This work was supported by grants from the Ligue Nationale Contre le Cancer and the Ligue Contre le Cancer Comité Isère, the Région Rhône-Alpes Génopole and Cancéropôle (CLARA).

\section{References}

1. Hartmann C, Boström J and Simon M: Diagnostic and molecular pathology of meningiomas. Expert Rev Neurother 6: 1671-1683, 2006.

2. Mantle RE, Lach B, Delgado MR, Baeesa S and Bélanger G: Predicting the probability of meningioma recurrence based on the quantity of peritumoral brain edema on computerized tomography scanning. J Neurosurg 91: 375-383, 1999.

3. Nordqvist AS and Mathiesen T: Expression of IGF-II, IGFBP-2, -5 , and -6 in meningiomas with different brain invasiveness. $J$ Neurooncol 57: 19-26, 2002.

4. Perry A, Stafford SL, Scheithauer BW, Suman VJ and Lohse CM Meningioma grading: an analysis of histologic parameters. Am J Surg Pathol 21: 1455-1465, 1997.

5. Pompili A, Derome PJ, Visot A and Guiot G: Hyperostosing meningiomas of the sphenoid ridge-clinical features, surgical therapy, and long-term observations: review of 49 cases. Surg Neurol 17: 411-416, 1982.

6. Perry A, Scheithauer BW, Stafford SL, Lohse CM and Wollan PC 'Malignancy' in meningiomas: a clinicopathologic study of 116 patients, with grading implications. Cancer 85: 2046-2056, 1999.

7. Louis DN, Scheithauer BW, Budka H, Von Deiming A and Kepes JJ: Meningiomas. In: Pathology and Genetics of Tumours of the Nervous System. Kleihues P (ed). IARC Press, Lyon, pp176-184, 2000

8. Riemenschneider MJ, Perry A and Reifenberger G: Histological classification and molecular genetics of meningiomas. Lance Neurol 5: 1045-1054, 2006

9. Carvalho LH, Smirnov I, Baia GS, et al: Molecular signatures define two main classes of meningiomas. Mol Cancer 6: 64 , 2007.

10. Cuevas IC, Slocum AL, Jun P, et al: Meningioma transcript profiles reveal deregulated Notch signaling pathway. Cancer Res 65: 5070-5075, 2005.

11. Fathallah-Shaykh HM, He B, Zhao L, et al: Genomic expression discovery predicts pathways and opposing functions behind phenotypes. J Biol Chem 278: 23830-23833, 2003.

12. Watson MA, Gutmann DH, Peterson K, Chicoine MR, Kleinschmidt-DeMasters BK, Brown HG and Perry A: Molecular characterization of human meningiomas by gene expression profiling using high-density oligonucleotide microarrays. Am J Pathol 161: 665-672, 2002.

13. Wrobel G, Roerig P, Kokocinski F, Neben K, Hahn M, Reifenberger $G$ and Lichter P: Microarray-based gene expression profiling of benign, atypical and anaplastic meningiomas identifies novel genes associated with meningioma progression. Int J Cancer 114: 249-256, 2005.

14. Durand A, Champier J, Jouvet A, Labrousse F, Honnorat J, Guyotat $\mathrm{J}$ and Fèvre-Montange M: Expression of c-Myc, neurofibromatosis Type 2, somatostatin receptor 2 and erb-B2 in human meningiomas: relation to grades or histotypes. Clin Neuropathol 27: 334-345, 2008 .

15. Pfisterer WK, Hendricks WP, Scheck AC, Nieman RA, Birkner TH, Krampla WW and Preul MC: Fluorescent in situ hybridization and ex vivo $1 \mathrm{H}$ magnetic resonance spectroscopic examinations of meningioma tumor tissue: is it possible to identify a clinicallyaggressive subset of benign meningiomas? Neurosurgery 61 1048-1061, 2007
16. Rempel SA, Ge S and Gutiérrez JA: SPARC: a potential diagnostic marker of invasive meningiomas. Clin Cancer Res 5: 237-241, 1999.

17. Tenan M, Fulci G, Albertoni M, et al: Thrombospondin-1 is downregulated by anoxia and suppresses tumorigenicity of human glioblastoma cells. J Exp Med 191: 1789-1798, 2000.

18. El Atifi M, Dupré I, Rostaing B, Chambaz EM, Benabid AL and Berger F: Long oligonucleotide arrays on nylon for large-scale gene expression analysis. Biotechniques 33: 612-618, 2002.

19. Champelovier P, El Atifi M, Mantel F, Rostaing B, Simon A, Berger $F$ and Seigneurin D: Effects of tumor necrosis factor- $\alpha$ $(\mathrm{TNF} \alpha)$ and interferon- $\gamma($ IFN $\gamma)$ on gene expression profiles in bladder carcinoma cells using oligonucleotide microarray analysis. Cancer Genomics Proteomics 1: 455-464, 2004.

20. Srivastava M, Torosyan Y, Raffeld M, Eidelman O, Pollard HB and Bubendorf L: ANXA7 expression represents hormonerelevant tumor suppression in different cancers. Int J Cancer 121: 2628-2636, 2007.

21. Sauer G, Kurzeder C, Grundmann R, Kreienberg R, Zeillinger R and Deissler H: Expression of tetraspanin adaptor proteins below defined threshold values is associated with in vitro invasiveness of mammary carcinoma cells. Oncol Rep 10: 405-410, 2003.

22. Jin SP, Kim JH, Kim MA, Yang HK, Lee HE, Lee HS and Kim WH: Prognostic significance of loss of $\mathrm{c}$-fos protein in gastric carcinoma. Pathol Oncol Res 13: 284-289, 2007.

23. Johansson T, Lejonklou MH, Ekeblad S, Stålberg P and Skogseid B: Lack of nuclear expression of hairy and enhancer of split-1 (HES1) in pancreatic endocrine tumors. Horm Metab Res 40: 354-359, 2008.

24. Park K, Kim K, Rho SB, et al: Homeobox Msx1 interacts with p53 tumor suppressor and inhibits tumor growth by inducing apoptosis. Cancer Res 65: 749-757, 2005.

25. Rossi M, Sayan AE, Terrinoni A, Melino G and Knight RA: Mechanism of induction of apoptosis by $\mathrm{p} 73$ and its relevance to neuroblastoma biology. Ann NY Acad Sci 1028: 143-149, 2004.

26. Shostak KO, Dmitrenko VV, Garifulin OM, et al: Downregulation of putative tumor suppressor gene TSC-22 in human brain tumors. J Surg Oncol 82: 57-64, 2003.

27. Tang D, Sivko GS and DeWille JW: Promoter methylation reduces C/EBPdelta (CEBPD) gene expression in the SUM-52PE human breast cancer cell line and in primary breast tumors. Breast Cancer Res Treat 95: 161-170, 2006.

28. Yamaguchi F, Morrison RS, Gonatas NK, Takahashi H, Sugisaki Y and Teramoto A: Identification of MG-160, a FGF binding medial Golgi sialoglycoprotein, in brain tumors: an index of malignancy in astrocytomas. Int J Oncol 22: 1045-1049, 2003.

29. Ganesan K, Ivanova T, Wu Y, et al: Inhibition of gastric cancer invasion and metastasis by PLA2G2A, a novel beta-catenin/TCF target gene. Cancer Res 68: 4277-4286, 200830.

30. Oka T, Ouchida M, Koyama M, et al: Gene silencing of the tyrosine phosphatase SHP1 gene by aberrant methylation in leukemias/lymphomas. Cancer Res 62: 6390-6394, 2002.

31. McPherson J, Sage H and Bornstein P: Isolation and characterization of a glycoprotein secreted by aortic endothelial cells in culture. Apparent identity with platelet thrombospondin. J Biol Chem 256: 11330-11336, 1981.

32. Bouamrani A, Ramus C, Gay E, et al: Increased phosphorylation of vimentin in noninfiltrative meningiomas. PloS One 5: e9238, 2010.

33. Sargiannidou I, Zhou J and Tuszynski GP: The role of thrombospondin-1 in tumor progression. Exp Biol Med 226: 726-733, 2001. 